\title{
Minimizing Gravity Sag of a Large Mirror with an Inverted Hindle-mount
}

\author{
David W. Robinson \\ NASA Goddard Space Flight Center, Greenbelt, MD
}

\begin{abstract}
A method of minimizing the optical distortion from gravity sag on a suspended large autocollimating flat mirror has been devised. This method consists of an inverted nine-point Hindle-Mount. A conventional Hindle-mount is located underneath api sky-viewing mirror and is primarily under compression loads from the weight of the mirror. It is not suitable for the situation where the mirror is viewing the ground, since a mirror would tend to fall out of the mount when in an inverted position. The inverted Hindle-Mount design consists of bonded joints on the backside of the mirror that allow the mirror to be held or suspended above an object to be viewed. This ability is useful in optical setups such as a calibration test where a flat mirror is located above a telescope so that the telescope may view a known optic.
\end{abstract}

Keywords: Hindle Mount Optical Mounting Techniques Bonding Epoxy Cell

\subsection{INTRODUCTION}

The optical design of the DCATT project called for a one-meter diameter flat mirror weighing 385 kilograms to be suspended above a telescope. The project desired the gravity sag on the figure of the mirror to be better than $\lambda / 15$ in the visible spectrum, or about 42 nanometers peak to valley deflection. This paper will describe the issues with mounting this large optic and the design that successfully achieved a low gravity sag by using an inverted Hindle-mount.

The DCATT (Deployable Comparative Active Telescope Testbed) was a ground-based project in support of the Next Generation Space Telescope (NGST) program. Its purpose was to provide a means to test wavefront sensing and control algorithms for use on NGST. The optical design of DCATT consisted of a one-meter diameter segmented primary mirror in a double-pass arrangement with an autocollimating (AC) flat mirror suspended above the telescope. As seen in Figure 1, the DCATT optical layout is unusual from typical optical systems in that the telescope only views the AC Flat, and not the night sky. 


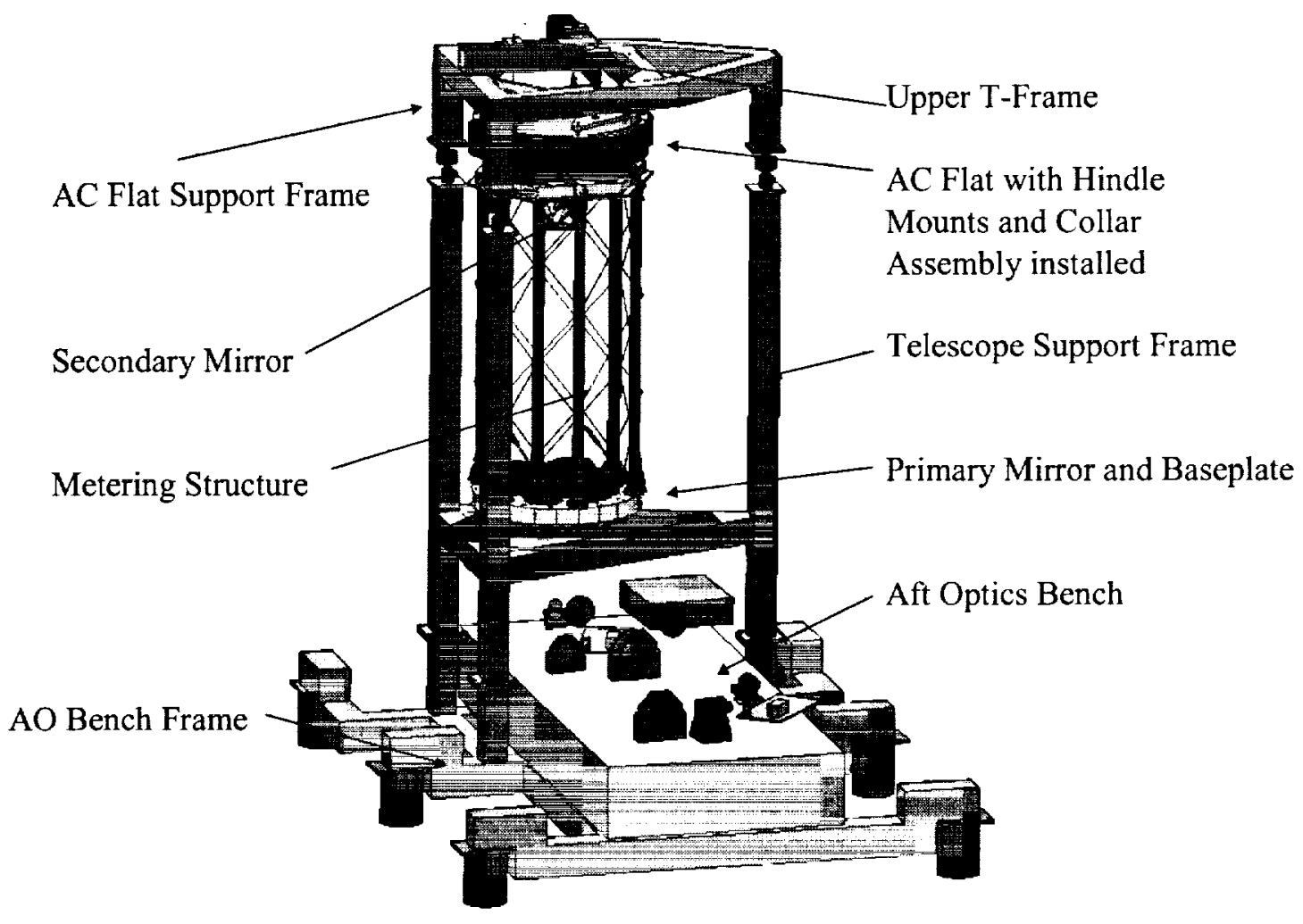

Figure 1. Schematic of the DCATT project. For more information, see http://dcatt.gsfc.nasa.govt

A well-characterized source of light was projected onto the AC Flat and the optical distortions caused by the telescope and aft optics were compared against it. In this fashion, various components and automated wavefront sensing and control features were tested.

\subsection{MINIMIZING GRAVITY SAG}

Hand calculations using Roark's formulas' and FEM analysis showed that simply supporting the AC Flat around the rim would cause a gravity sag of about 354 nanometers within the $92 \mathrm{~cm}$ usable aperture of the mirror, which is about an order of magnitude higher than the requirements allowed. The sag pattern is shown below in Figure 2. To meet requirements, the mirror would have to be constrained in a more elaborate way. After considering several alternatives, the project decided to use a Hindle-mount.
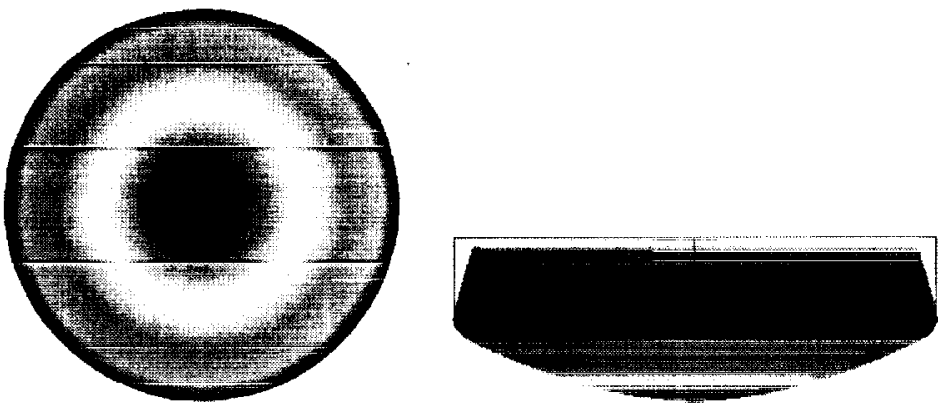

Figure 2. Front and side view of gravity sag from simply supported mirror (highly distorted for clarity) 
A Hindle-mount is a multiple-point mounting scheme first described by Dr. J.H. Hindle in a classic 1945 paper $^{2}$ and further described by Yoder ${ }^{3}$, and others. The mount relies on precise location of equally loaded support points to minimize the sag. Three, six, nine, eighteen, and other multiples of three are possible. As the number of mounting points increases, the sag reduces proportionately.

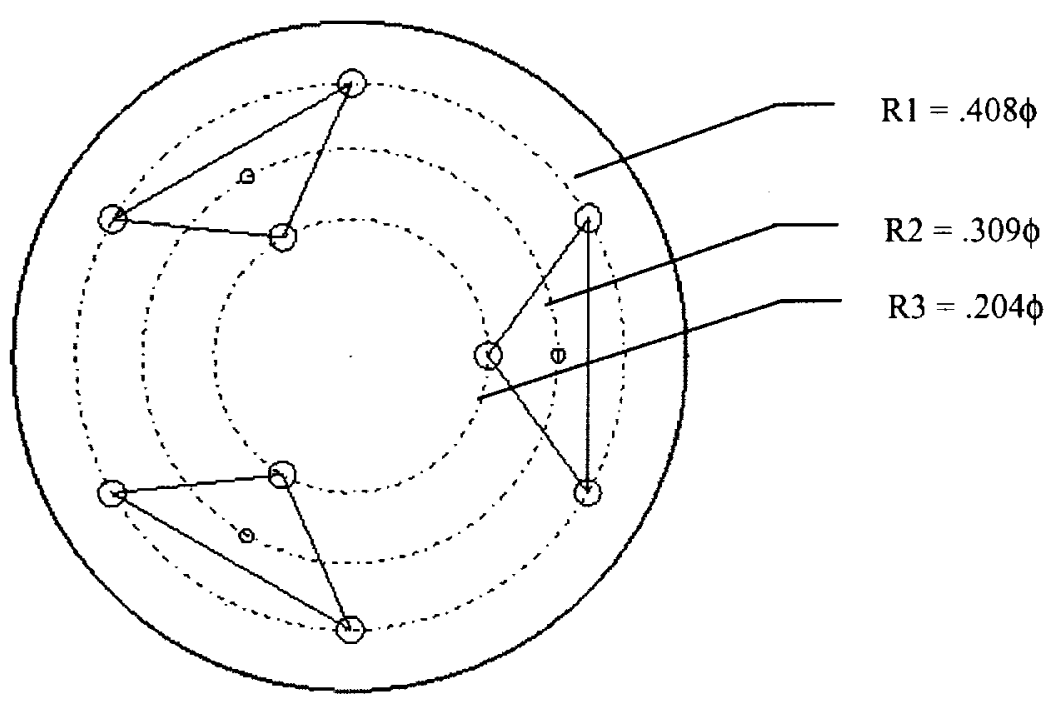

Figure 3. Schematic of a 9-point Hindle-mount, from Mehtat.

As shown in Figure 3, the nine point Hindle-mount consists of 6 mounting points on a circle with a radius of .408 of mirror's diameter, and three inner mounting points on a circle with a radius of .204 of the diameter. Groups of three points are connected to a plate having its centroid on a circle with radius .309 of the mirror's diameter. When the three triangle plates are picked up at their centroids, equal stress is applied to all nine points.

In Hindle's paper, the mount was designed to support a mirror looking up, with its reflective side facing the sky and the backside is resting on the mounting pads. As mentioned earlier, the DCATT application required the AC Flat to look down at the telescope. In order to do this, the Hindle-mount had to be inverted and the mirror suspended from the mounting pads.

\subsection{Gravity Sag Analysis}

A finite element analysis on the DCATT AC Flat showed that a nine-point Hindle-mount would cause a gravity sag of 49 nanometers (peak to valley) within the aperture visible to the telescope ${ }^{5}$. Although the gravity sag goal was 42 nanometers, the project accepted this as close enough.

The ideal Hindle-mount has infinitely small point contacts on the mirror. In reality, each contact point must have some surface area for bonding the mounting pad, or anchor, to the backside of the mirror. A finite element analysis was performed to determine how the surface area of the bond affected the gravity sag $^{6}$. A comparison was made with 1 square inch surface area bonds and 3 square inch bonds. The results were that the peak to valley gravity sag would not significantly change with an increased bond area. The predicted sag was 49 nanometers, peak to valley. 


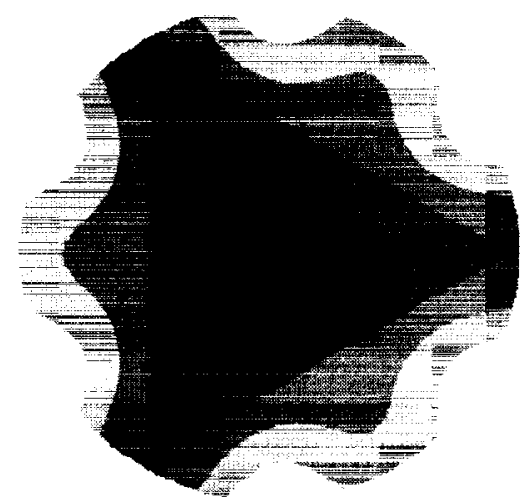

Figure 5. Mirror bottom surface deflection pattern

\subsection{CELL DESIGN}

Once the inverted Hindle-mount concept was settled upon, it had to be integrated into the design of the handling fixture, or cell, of the mirror, as well as the larger support frames that would ultimately hold the mirror in place above the telescope.

The mirror was made from Zerodur $®$ low expansion glass with a diameter of 101.7 centimeters and a thickness of 18.8 centimeters with a weight of $385 \mathrm{~kg}$. When the raw uncoated Zerodur blank arrived at the NASA Goddard Space Flight Center, it was sitting on a wooden pallet in a large plywood shipping container. We designed and fabricated a cell to fit around the mirror for handling. The cell was named the "collar assembly" and is shown in Figure 6. It consisted of four semi-circular arcs of aluminum, called "ring segments" that bolted around the sides of the mirror. Four brick-like blocks spaced 90 degrees apart around the circumference of the mirror connected the ring segments and provided additional hard points on the side of the collar that were useful when rotating the mirror from shiny side up to shiny side down. The total mass of the collar assembly was about $50 \mathrm{~kg}$, but no single piece weighed more than $10 \mathrm{~kg}$, so it was easy for one or two people to install.

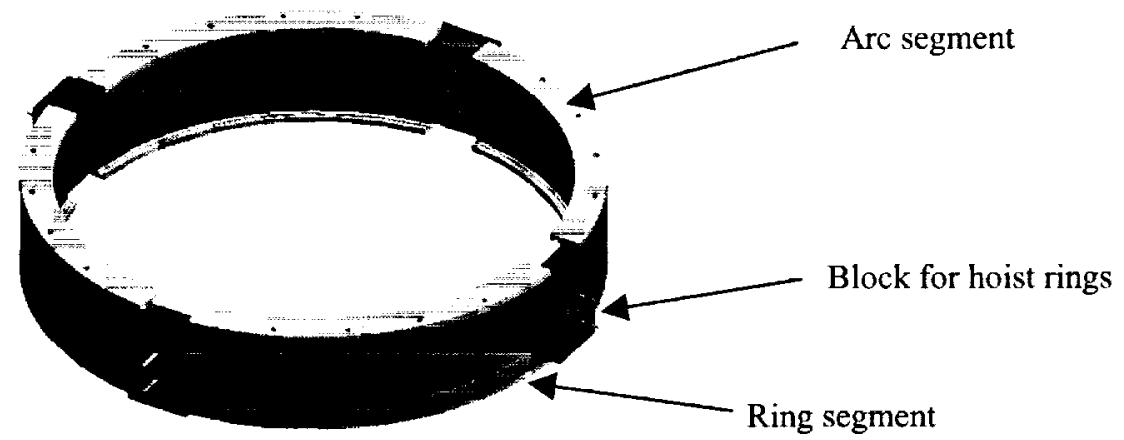

Figure 6. The Collar assembly

Eight flanges, or arc-segments, served to capture the AC Flat and contain it within the ring segments. Each arc-segment had a viton o-ring of 0.25 inches diameter in a groove running along the arc, shown in Figure 7. The AC Flat rested on these o-rings and never touched the aluminum components. 


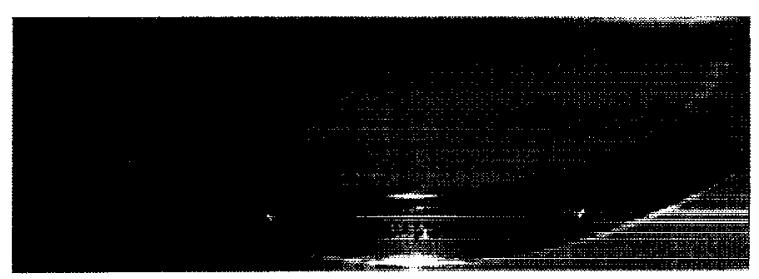

Figure 7. One of eight arc segments showing the viton o-ring that contacts the mirror surface

To prevent the mirror from sliding and shifting within the collar assembly, four pairs of setscrews with rubber tips were installed into the comer blocks. Each cormer block also had a leveling pad, or foot, screwed into its base so that the AC Flat and collar assembly could be set down on the floor, as shown in Figure 8.
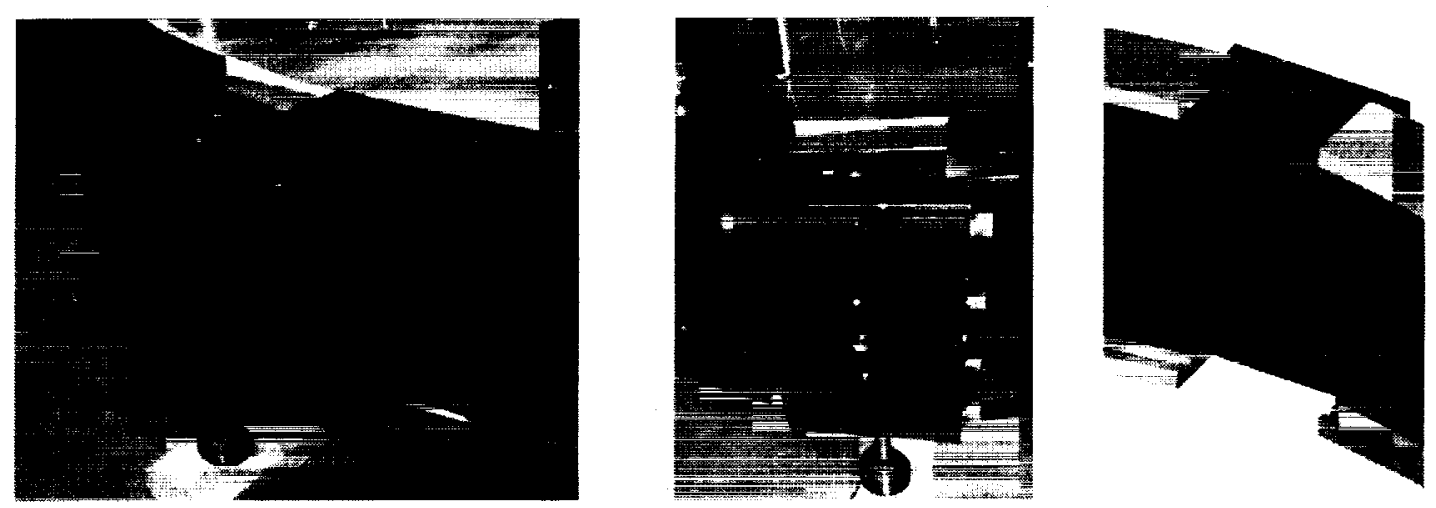

Figure 8. Views of corner block showing hoist ring locations, leveling pad foot \& pusher pads

To mount the AC Flat into the DCATT assembly, the top four arc-segments were removed from the collar, and the collar was then bolted directly to the AC Flat Support Frame shown in Figure 10. The AC Flat Support Frame was stacked onto the Telescope Support Frame that supported the telescope (Figure 1). The arc segments were removed to allow the mirror to be lifted up a centimeter or so by the Hindle-mounts but yet still remain within the collar assembly. This provided a critical safety feature since if the Hindlemounts failed for any reason, the AC Flat would simply tilt or settle back into the collar assembly without damage and danger to the telescope and personnel below.
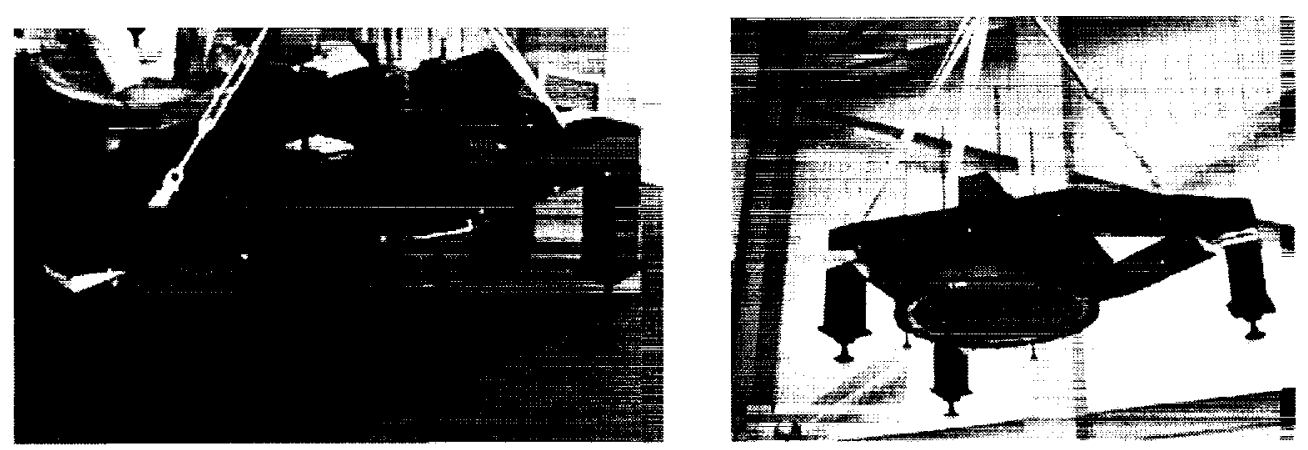

Figure 10. Views of the AC Flat Support Frame with mirror simulator installed 


\subsection{HINDLE MOUNT DESIGN}

In an attempt to avoid re-inventing the wheel, the team performed a literature search of Hindle-mounts and several papers were found discussing the use of Hindle-mounts. Unfortunately, none were found that described an inverted Hindle-mount, and particularly how the mounting pads could be attached to the mirror. This led the project to initiate a development effort.

The DCATT Hindle-mount design consists of three sets of three mounting points, called anchors. The anchors are made from Invar, and a hole is tapped into them to allow a triangular plate to be attached with bolts. The anchors are bonded to the mirror in specific locations with epoxy. Choosing the right epoxy and preparation techniques required a development effort that is described later. Each set of three anchors is bolted to a one-inch thick aluminum triangular plate, and a spherical washer set was placed under each bolt head. The plate is picked up by a clevis inserted into a rod-end at the centroid of the triangle formed by the three points, as shown in Figure 11. The clevis is connected to a threaded rod that goes through the AC Flat Support Frame as shown in Figure 12.

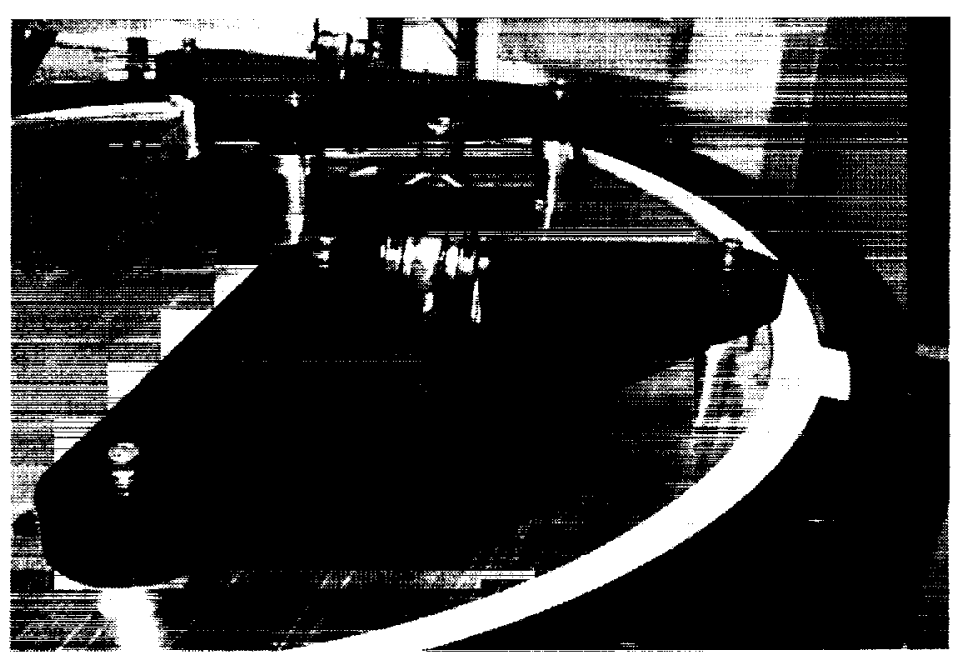

Figure II. View of two Hindle-mounts, showing clevis and rod ends

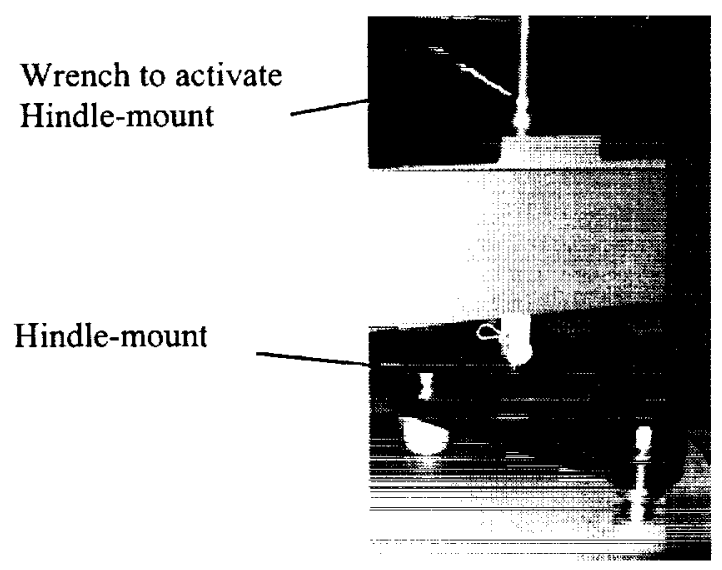

Figure 12. Hindle-mount with triangular plate, bonded anchors, and activation stud through upper frame

Activating the Hindle-mount was a simple matter of tuming the nut connected to the long threaded rod. Near the top of Figure 12, a wrench can be seen on this nut. Turning it lifted the mirror up from the collar 
assembly. To ensure a vertical lift from the collar assembly using three Hindle-mounts, the process is to turn each of the three nuts a quarter-tum in sequence until the desired mirror elevation and orientation is obtained. Typically the mirror was raised about $2 \mathrm{~mm}$ from its resting position on the arc-segment o-rings.

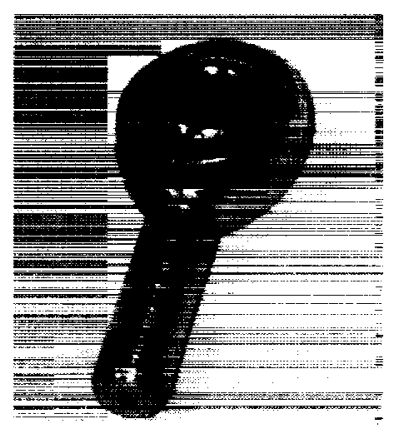

Figure 13. Typical rod-end

A key aspect of the Hindle-mount mechanism is the ball-joint rod-end located at the centroid of the triangle plate. As seen in Figure 13, a rod-end is an eyebolt with a swiveling insert that is free to rotate in all 3 rotational degrees of freedom. This feature eliminates the possibility of putting bending moments into the bonded joints of the Hindle-mount. Bending moments are to be avoided since they place the bond areas in peel, and even small peel loads risk breaking the epoxy bonds.

Experience showed that it was better to keep the bolts on the anchors untorqued so that the triangle plate was free to shift slightly on the anchors. If the plate were securely fastened to the anchors, reaction forces in the form of peel loads would be created at the bond sites due to the slight bending (about .0003") of the plate as it becomes loaded with the weight of the mirror.

\subsection{BOND DESIGN AND TESTING}

The largest problem in this Hindle-mount design was how to connect the Hindle-mount pads to the glass surface. Many engineering hours could have been saved if one could drill and tap glass. The only reasonable solution appeared to be bonding the mount pads to the glass with epoxy.

Two epoxies were selected for tensile testing, based on a survey of vendors and on-site experts. The two epoxies were Stycast 2850 and 3M's 2216 clear epoxy. Both of these products have a long heritage at NASA. We decided to make the anchors from Invar, a very low expansion metal. Using metal instead of glass for the anchor would simplify connection to the triangular plate of the Hindle-mount since the metal could be tapped and bolted to it.

Test specimens were made, bonded and cured per manufacturer's instructions to $2 \times 2 \times 1$ "Zerodur blocks. The anchors were pulled from the substrate blocks until failure in an Instron 1125 Universal-testing machine. The expected service load was only $94.4 \mathrm{psi}$. All of the samples had a tensile bond strength over 800 pounds per square inch. To make an informed choice, the failure modes were examined. All of the Stycast 2850 samples failed by pulling a chunk of the substrate out while all of the 2216 epoxy samples failed in adhesion to the substrate. The 2216 epoxy was chosen since it had a more benign failure mode. The tensile bond strengths for these two Invar/2216 test samples were 1396 and $1688 \mathrm{psi}^{7}$.

\subsection{First Attempts at Bonding}

Once the anchor material and design was settled upon, a significant problem arose as to how to locate the anchors on the backside of the AC Flat for the bonding process. The allowable error in position was about .060 inches. Position error greater than this would prevent attachment of the triangular plate. A metal 
template was fabricated to which the anchors were bolted, as shown in Figure 14. The anchors were then coated with epoxy and the entire template was laid up on the mirror.

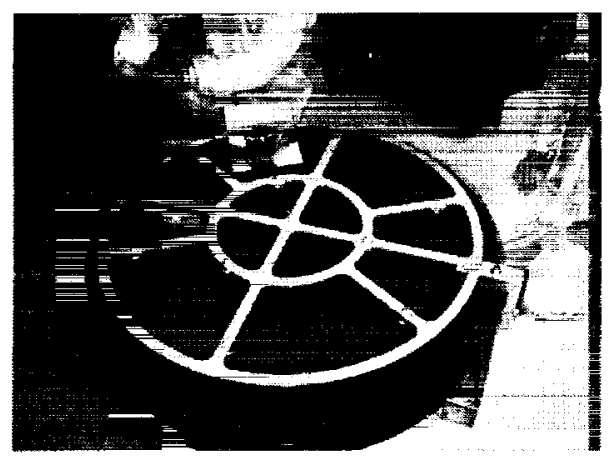

Figure 14. Bond template locating the position of the nine Invar anchors on the mirror

This design did not work well since the template was too flexible and did not allow enough individual control of the bondlines. A better solution was to use the template as a stencil to mark where the nine anchors were to be bonded. Then each bond was made one at a time. A triangular plate, shown in Figure 15 , was used to hold a group of three anchors in place while the bond cured. The final position error was estimated at about .030 inches per anchor.
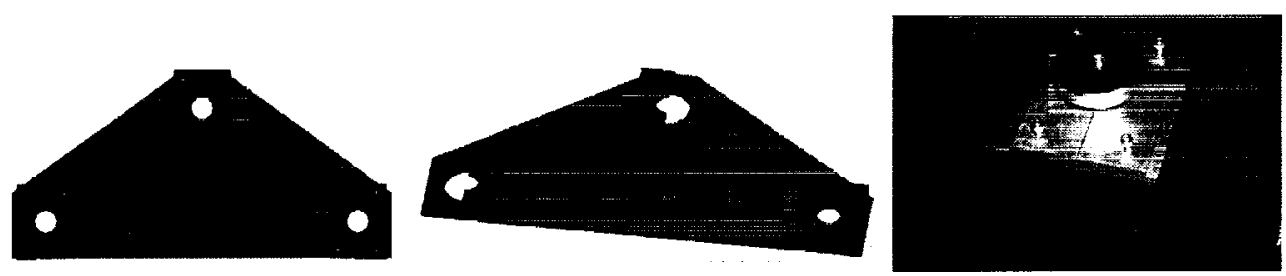

Figure 15. Views of the template that held the anchors in place during epoxy curing

\subsection{Bond Endurance Problems}

In mid 1999, the Hindle-mounts were successfully activated and left untouched in the corner of a temperature and humidity controlled cleanroom. Sometime between 6 and 8 weeks later, one of the bonds failed unexpectedly. The collar assembly cell around the mirror prevented any damage from occurring to the mirror.

While the failure investigation was ongoing, the anchor was re-bonded and the Hindle-mounts re-activated. Unfortunately, a failure occurred on a different anchor the following week. A thorough investigation of the failures by NASA materials experts failed to determine the cause. Several papers in the adhesives literature indicated that initial bond strength had no correlation to bond endurance, and that in general, tensile testing provides little information about how long the bond would hold in service.

\subsection{Efforts to Improve Bond Endurance}

The basic approach to solving the problem was to reduce tensile stress on the bond, prepare the bond site more carefully, and test. The anchor footprint was tripled, to increase bond surface area from 1 to 3 square inches. This reduced the average stress on the bond from 94.4 to $31.5 \mathrm{psi}$. Bond site preparation changes included using methylene chloride to clean the bond area, and upon the advice of $3 \mathrm{M}$, using 3M's 3901 primer prior to bonding. Microspheres of .003 inches diameter were added to the 2216 epoxy to control the bondline thickness. Also, the bond was allowed to cure at a slightly elevated temperature $(110 \mathrm{~F})$ by blowing warm air on it for 7 days. This accelerated the curing process. 
After these steps were taken, the Hindle-mounts were reactivated and have been holding since October 1999. Since the cause of the previous bond failures were not well understood, the epoxy joints are naturally viewed with some skepticism. Without a better understanding of the adhesive physics and the ability to predict lifetime, the project is relying on long-term testing in service.

\subsection{ALTERNATIVE TO INVERTED HINDLE MOUNT}

Interestingly, as the project was approaching completion, DCATT engineers became aware of a technique demonstrated by Kodak in Rochester, New York. In Kodak's application, they too had a 1-meter diameter optic that had to be very flat in the inverted position. Kodak simply inverted the mirror and used the ionbeam milling process to remove the Zerodur material as necessary to achieve the specified flatness. When oriented with the shiny side up, the mirror has a tiny parabolic curve. When the mirror is inverted, the curve straightens out and the surface becomes flat. This technique allows the mirror to be simply supported around its rim, greatly simplifying the mounting scheme and eliminating the need for a Hindle-mount. Any future projects faced with mounting a large inverted optic should consider both the ion-beam milling process and the Hindle-mount design.

\subsection{CONCLUSIONS}

A nine-point Hindle-mount has been shown to work in an inverted position for a heavy optic, and the gravity sag has been shown by analysis to be about an order of magnitude less than that caused by supporting the optic around the perimeter. One disadvantage is that obtaining reliable epoxy bonds between the Hindle-mounts and the mirror can be difficult. Safety features should be installed into the

design of the handling fixtures such that a failure of the epoxy bonds will not cause the mirror to fall. Long duration epoxy bond testing may need to be incorporated into a project's schedule.

\section{ACKNOWLEDGEMENTS}

This work could not have been done without the participation of many talented people on the DCATT team and at NASA Goddard. The author would particularly like to thank Claudia LeBoeuf, Pam Davila, Mike Wade, Mike Viens, John Carro, and Matt Fenske for their advice, wisdom, and patience.

\section{REFERENCES}

1. Young, Warren, Roark's Formulas for Stress \& Strain, p. 429, McGraw-Hill, New York, 1989.

2. Hindle, J.H. "Mechanical Flotation of Mirrors," in Amateur Telescope Making, Book One, A. G. Ingalls, Ed., p. 229, Scientific American, 1945.

3. Yoder, Paul, R. Opto-Mechanical Systems Design, $2^{\text {nd }}$ edition, p. 302, Marcel Dekker, Inc., 1993.

4. Mehta, P. K., "Flat circular optical elements on a 9-point Hindle-mount in a l-g force field," p. 118 , Proceedings of SPIE 450, 1983.

5. Carro, John. Internal NASA GSFC memo, dated 9/1/99.

6. Ibid.

7. Viens, Michael, NASA GSFC internal memo dated $4 / 8 / 98$. 